\title{
Los programas de fomento a la innovación para pymes en la encrucijada de la discontinuidad administrativa
}

\author{
Programs to innovation for smes at the crossroads of the administrative discontinuity \\ Ismael Acevedo-Sánchez ${ }^{a}$, Reyes Acevedo-Sánchez ${ }^{b}$, Alfredo Castillo-Trejo $^{c}$
}

\begin{abstract}
:
This article describes the position of the following federal administration in relation to the National Council of Science and Technology (CONACYT), to continue with the Program for the Promotion of Innovation (PFI) or to change to other types of strategies that foster the stimulus to innovation by the private sector, this situation is part of the debate that has been worked on administrative discontinuity (Peña, 1999), this concept is to ensure the institutional growth programs to transcend beyond the six-year terms. It is important to emphasize the subject of innovation of SMEs, because according to the Latin American and Caribbean Economic System SELA (2009), SMEs constitute more than $90 \%$ of companies in most countries of the world. In Latin America, empirical studies estimate that these contribute between $35 \%$ and $40 \%$ to the generation of employment in the region, $33 \%$ of GDP and $25 \%$ of investment. On the other hand, the Ministry of Economy in Mexico (2008), corroborates these results, to infer that in this area there are about 17 million formal companies, 95\% microenterprises and 1.5 million small and medium, which contribute $40 \%$ of the employment, $33 \%$ of GDP and $25 \%$ of investment. Therefore, it is necessary to resume the projects related to the innovation of SMEs that have been developed in the administration of Enrique Peña Nieto and especially those that are contemplated in the National Development Plan of Andrés Manuel López Obrador.
\end{abstract}

Keywords:

Administrative discontinuity, Innovation, Program, Plan.

Resumen:

El presente artículo describe la postura de la siguiente administración federal con relación al Consejo Nacional de Ciencia y Tecnología (CONACYT), de continuar con el Programa de Estímulo a la Innovación (PFI) o cambiar a otro tipo de estrategias que permita fomentar el estímulo a la innovación por parte del sector privado, esta situación forma parte del debate que se ha trabajado en la discontinuidad administrativa (Peña, 1999), esté concepto plantea asegurar los programas de crecimiento institucional para que trasciendan más allá de los sexenios. Es importante hacer énfasis en el tema de innovación de las PYMES, porque de acuerdo con el Sistema Económico Latinoamericano y del Caribe SELA (2009), las PYMES constituyen más del 90\% de las empresas en la mayoría de los países del mundo. En América Latina, estudios empíricos estiman que éstas contribuyen entre 35\% y $40 \%$ a la generación de empleo en la región, 33\% del PIB y 25\% de la inversión. Por otra parte, la Secretaria de Economía en México (2008), corrobora estos resultados, al inferir que en esta zona existen cerca de 17 millones de empresas formales, $95 \%$ microempresas y 1,5 millones pequeñas y medianas, que aportan $40 \%$ del empleo, $33 \%$ del PIB y $25 \%$ de la inversión. Por lo tanto, es necesario retomar los proyectos con relación a la innovación de PYMES que se han desarrollado en el sexenio de Enrique Peña Nieto y sobre todo los que están contemplados en el Plan Nacional de Desarrollo de Andrés Manuel López Obrador.

Palabras Clave:

Discontinuidad administrativa, Innovación, Programa, Plan.

\section{Introducción}

Las políticas públicas deben establecerse en virtud de beneficiar a la mayor cantidad de población posible, buscando siempre el bienestar colectivo independientemente del partido que este en el poder, el presente artículo plantea una reflexión con relación a la discontinuidad administrativa que se da cada sexenio, lo cual deja inconclusos algunos proyectos que no fueron bien entendidos por las administraciones entrantes. El programa de Fomento a la Innovación en la PYMES es uno de esos proyectos que ha desaparecido y que

a Autor de Correspondencia, Profesor de Tiempo Completo de la Universidad Tecnológica de Tula-Tepeji. E-mail: ismael.acevedo@uttt.edu.mx

b Profesor por asignatura B del Tecnológico de Estudios Superiores de Jilotepec. E-mail: acevedo333@ yahoo.com.mx

c Profesor de Tiempo Completo de la Universidad Tecnológica de Tula-Tepeji. E-mail: Alfredo.castillo@uttt.edu.mx 
dejaran sin apoyo a una gran cantidad de pequeñas empresas que empezaban a despuntar, gracias al apoyo de este tipo de programas por medio del Conacyt.

\section{Metodologia}

Para el desarrollo de éste artículo se utilizó una investigación exploratoria de tipo documental, la cual abarcó la descripción y explicación del fenómeno que se investiga, con el propósito de describir la situación que se presenta en el cambio de la administración pública a fin de evaluar diversos aspectos, dimensiones y componentes del fenómeno a estudiar (Hernández et. al., 2010), en este tipo de estudio se miden conceptos o variables relacionadas con la discontinuidad, es decir como es, y se manifiesta el fenómeno de interés centrado en medir con la mayor precisión posible (Selltiz, 1965). Se considera que este articulo ofrece la posibilidad predicción, aunque sea rudimentaria ya que influirá en la opinión pública.

\section{Estado del Arte}

Con base del desarrollo de esta investigación se responden las preguntas originales basadas en la metodología que se señaló, justificando argumentos que se presentan a continuación; con relación a la innovación de la PYMES en México, es necesario mejorar el entorno económico y el apoyo directo a las empresas con instrumentos que generen las condiciones que contribuyan a su establecimiento, crecimiento y consolidación; no obstante muchas PYMES no logran desarrollarse o consolidarse, su tasa de éxito se encuentra por debajo de la media mundial con valores alrededor de los 25 y $30 \%$, contra un $40 \%$ que representa esta media; se han identificado una serie de elementos por los cuales esta empresas suelen fracasar dentro de los cuales los de mayor influencia son; financiamiento, falta de asesoría empresarial, deficiente administración, recursos humanos no calificados, desconocimiento de mercados, desconocimiento de la tecnología e innovación, además de una deficiente organización; todos estos factores contribuyen a una falta de competitividad de las PYMES en nuestro país. Es necesario apoyar a estas entidades económicas a superar los diversos obstáculos con los que se enfrentan para su crecimiento y competitividad; como la falta de una estructura productiva, el acceso a los créditos, la falta de una cultura de conocimiento, la poca innovación hacia los clientes y proveedores, el acceso y utilización a la tecnología, además de la falta de certificaciones de calidad, de acuerdo con datos de la Secretaria de Economía (2011), solo el $24 \%$ es dueña de alguna patente o licencia, por lo tanto la tecnología es un factor que puede jugar a favor o en contra de las PYMES.

Góngora y Madrid (2010), señalan que las actividades de Investigación, Desarrollo e Innovación (I+D+i) deben reforzarse para mejorar la ventaja competitiva de las PYMES, respecto a la innovación, ésta puede presentar diferentes formas, como la creación de nuevas tecnologías y conocimientos para mejorar los procesos y tecnologías y conocimientos creados en otra parte para desarrollar el desempeño económico de regiones y empresas.

De acuerdo con Dini y Stumpo (2011), la situación de rezago de la gran mayoría de las PYMES hace que una de las áreas de acción que tendría que adquirir una cierta relevancia, está relacionada con el fomento a la innovación y modernización tecnológica. En efecto en muchos países de Latinoamérica, en los últimos años, ha habido avances en las políticas de Ciencia y Tecnología (СуT). Sin embargo, estos cambios, que han llevado a modificaciones en la institucionalidad y en los enfoques de las intervenciones, no parecen haber llevado a procesos de modernización tecnológica sustantivos de la mayoría de las PYMES. Esto lleva a la necesidad de analizar las políticas, los instrumentos, las modalidades de intervención y las características de la institucionalidad que está orientada a la promoción de la innovación y modernización tecnológica de las firmas de menor tamaño.

La CEPAL (2011) señala que existen tres elementos relevantes que deberían ser considerados tanto en el diseño de las políticas como en la realización de investigación sobre PYME e innovación:

1. Las PYMES adoptan estrategias de innovación (que denominamos informales) distintas de las que desarrollan las grandes empresas. La eficacia de dichas estrategias depende del contexto competitivo en que operan las firmas.

2. La capacidad de las PYMES de relacionarse de forma eficiente con su entorno económico e institucional incide de forma muy significativa sobre sus estrategias informales de innovación.

3. El acceso de las PYMES a procesos más formales de innovación puede ser facilitado impulsando su vinculación con actores económicos que no experimentan las limitaciones de escala que afectan a estas empresas.

La innovación es un proceso social complejo que puede alcanzar distintos grados de complejidad y formalización. En particular, las empresas realizarían inversión en I+D una vez que han comprobado que el esfuerzo informal que inicialmente desarrollan ya no es suficiente para alcanzar los objetivos esperados de mejora en productividad o competitividad. Sobre esta base Katz (2011), reconoce que PYMES y grandes empresas tienen ventajas y desventajas diferentes y complementarias para la promoción de la innovación: las grandes empresas cuentan generalmente con mayores recursos (en dinero y conocimientos), mientras que las PYMES tienen "ventajas de comportamiento" relacionadas con la organización interna de la producción, que facilita la participación activa de los trabajadores, una comunicación más fluida entre las personas involucradas y la rapidez en la toma de decisiones, entre otras, en este sentido hay que tomar en cuenta que, dadas las especificidades del proceso de innovación en las PYMES, 
habría que orientar sus acciones hacia la utilización de instrumentos que permitan mejorar la capacidad de estas firmas de relacionarse con las grandes empresas, de participar de redes productivas, de establecer vínculos con el entorno social y productivo local. Por lo tanto, en el caso de las PYMES estos tipos de acción tendrían que adquirir mayor importancia en comparación con los incentivos directos para incrementar el gasto en I+D, como lo señala en el PEI, Es decir que hay un conjunto de instrumentos que se pueden llamar "indirectos" porque su objetivo principal no es fomentar la innovación y modernización (sino crear redes o desarrollar territorios); pero estos mismos instrumentos pueden inducir un conjunto de cambios importantes al interior de las firmas (introducir nuevas tecnologías, generar un mayor intercambio de información, promover formas de colaboración con instituciones y otras firmas que vayan modificando el modus operandi de la empresa). Por lo tanto, el PEI que el CONACYT mantienen con las PYMES debiera continuar a pesar del cambio del nuevo gobierno.

Con relación a la discontinuidad administrativa, se puede encontrar, cuando en una organización pública se presenta (por diversas razones) un cambio o no de su titular, quien tiene a cargo el proceso de toma de decisiones, el cual impacta directa o indirectamente, tanto al interior como al exterior de la organización. A este respecto Peña (1999) plantea; [...], estamos acostumbrados a que, en cada sexenio, haya cambios en el gobierno mexicano de actores que ocupan puestos de mandos superiores como son los secretarios de estado, sin que esos cambios sean para eficientar la administración pública, lo sorprendente sería que en esos puestos hubiera continuidad, pareciera ser que el método de ensayo-error fuera el más aceptado por nuestros gobernantes.

Con relación al PEI del CONACYT, Enrique Cabrero ex Director del Concejo, enfatizó la importancia de que un proyecto con visión a largo plazo se estimule la innovación generada por la industria junto con la academia y que este proyecto perdure más allá de la transición gubernamental como se ha señalado anteriormente.

\section{Conclusión}

Los programas federales que tengan como meta el desarrollo de los sectores productivos del país debería de perdurar a pesar de la transición de las nuevas administraciones, no se trata de hacer "un borrón y cuenta nueva" sino de analizar lo que sea mejor para el colectivo nacional, tampoco se trata de colocarse medallas de manera individual o por sexenio, se trata de engrandecer a este gran país llamado México y al final eso es lo que cuenta. De acuerdo con Vega (2016) [...] los "nuevos" gobiernos en sus campañas políticas ofrecen cambios radicales y actuaciones más eficientes en su desempeño, éstos vuelven a colocar en puestos directivos estratégicos a actores decisores de las anteriores administraciones. Con base en esto se puede concluir que las instituciones prevalecerán siempre por encima de las personas y los resultados de los programas dependerá de la gestión del personal en turno, por lo que la discontinuidad deberá cambiar a una continuidad en procesos y programas que mejoren las condiciones y vida de los ciudadanos de este país.

\section{Referencias}

Dini M. y Stumpo G. (2011) Políticas para la innovación en las pequeñas y medianas empresas en América Latina. Chile. CEPAL

Góngora G. y Madrid A. (2010) El apoyo a la innovación de la PYME en México. Un estudio exploratorio. Investigación y Ciencia. Universidad Autónoma de Aguascalientes. Núm. 47 (21-30).

Hernández, R., Fernández, C. y Baptista, P. (2010) Metodología de la investigación. México, Ed. McGraw Hill.

Jorge Katz, J. (2011) Políticas para la innovación en las pequeñas y medianas empresas en América Latina. Chile. CEPAL

Peña C., M. P. (1999). Discontinuidad administrativa en el sector público: el caso del gobierno federal mexicano en el período 1988-1998. Tesis doctoral, Instituto Politécnico Nacional, Distrito Federal (D.F.), México.

Plan Nacional de Desarrollo 2013-2018 (2013) Programa de desarrollo Innovador 2013-2018. México. Gobierno de la República. Consultado el 17 de febrero del 2017. Disponible desde internet en:

http://www.economia.gob.mx/files/prodeinn/Programa de Des arrollo Innovador2013-2018.pdf

Secretaria de Economía (2011). Contacto PyME. Consultado el 8 de marzo del 2015. disponible desde internet en: http://www.economia.gob.mx/swb/es/economia/p Contacto P yME

Sistema Económico Latinoamericano y del Caribe SELA (2009). Políticas e instrumentos para enfrentar el impacto de la crisis económica internacional sobre las Pequeñas y Medianas Empresas (PYMES) de América Latina y el Caribe. Secretaría permanente del SELA. Caracas, Venezuela. Mayo SP/Di N $06-09$.

Vega, M. (2016) Discontinuidad vs. Continuidad administrativa en organizaciones públicas: una perspectiva preliminar para el análisis organizacional. Revista DOXA Volumen 5 / Número 10 / Primer Semestre 2016. Disponible desde internet en: http://doxa.uach.mx/assets/r10 articulo12.pdf 